\title{
American Public University System Observatory
}

\author{
Ed Albin
}

American Public University System

The American Public University System observatory is located on the main
center campus in Charles Town, West Virginia, standing atop the tallest
building on the site with a bright, silver 22 and a half feet wide dome. Within its protective cavern the CDK 24 inch diameter telescope is housed, mounted on a PlaneWave, A200 equatorial pier that stands at an overall height of 10 feet. There are not one but two telescopes attached to the central pier, with a 5 inch diameter Tele Vue refractor fastened on top of the 24 inch using additional brackets. Both telescopes use an SBIG type of CCD camera, the CDK uses a model ST 16803 and the Tele Vue uses an ST 8300, each with full set filter wheels for different research and photographic needs. The power of this set up lies in its equipment as much as its remote operations capabilities, wherein faculty, professors and even students may access and control the telescopes from vastly different and far away locations in pursuit of any astronomical objects.

There are several objects that have been or are continuing to be observed and projects carried out with the APUS observatory, including variable stars, nebulae, galaxies, exo-planets, supernovae, and star clusters. Some of the research conducted has been part of an ongoing effort, like the supernova search project, led by Dr. Kristen Miller, where weekly or biweekly images are obtained of over 140 different galaxies and made easily accessible to volunteering students to look over as they analyze them for any newly occurring transients as possible supernovae. There are 18 volunteers actively searching these images and reporting their results, which allows for open access to real data for the students and a way to enable greater learning and science. Another project involved taking photometric images of several variable stars and potential variable stars as highlighted by the American Association of Variable Star Observers (AAVSO). This project involved taking images and analyzing them using open software hosted by the AAVSO as well as installed on the APUS observatory suites to measure star magnitudes and report them to the variable star community. The resultant light curves is currently being analyzed and used for a paper in the APUS SPST690 (Independent Study) class, and the data is now readily available for other observers to work with or include in future research. The observatory has also participated in efforts to track and detect new asteroids, which is an ever-growing part of the space science fields, for both earth security measures and increasing science goals of understanding the ancient building blocks of the solar system. Other such solar systems are also of interest by the faculty and staff at APUS, as they use the observatory to image 
known stars hosting exoplanets and add transit data to the scientific community for continuing research.

Furthermore, beautiful photographic works have been undertaken at APUS using the CCD cameras and installed software to image and process several nebulae, galaxies and more. A listing and brief description of some color processed images composed with the APUS CDK 24-inch telescope can be found below.

The Horsehead Nebula in the constellation Orion is a dark and emission nebula dominated by hydrogen gases. This shot was taken with the combination of several R, G, and B filtered images each taken at 180 seconds and stacked together.

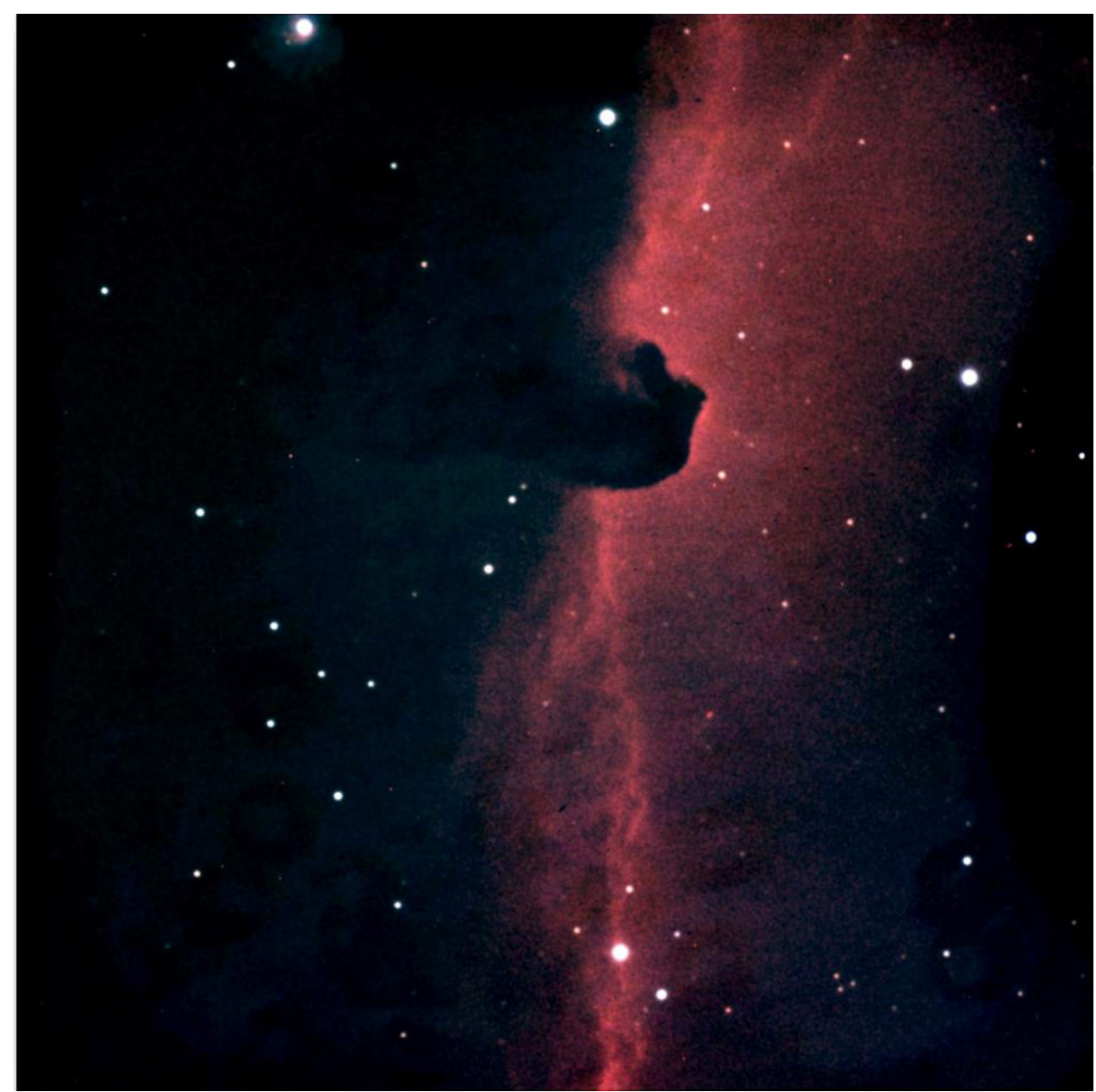

Figure 1. The Horsehead Nebula 
The Rosette Nebula is a vast nebula that only partially fit in the field of view of the CDK 24-inch telescope. Nevertheless, its rendering is seen here, also taken with the R, G and B filters and 180-second exposures and later stacked.

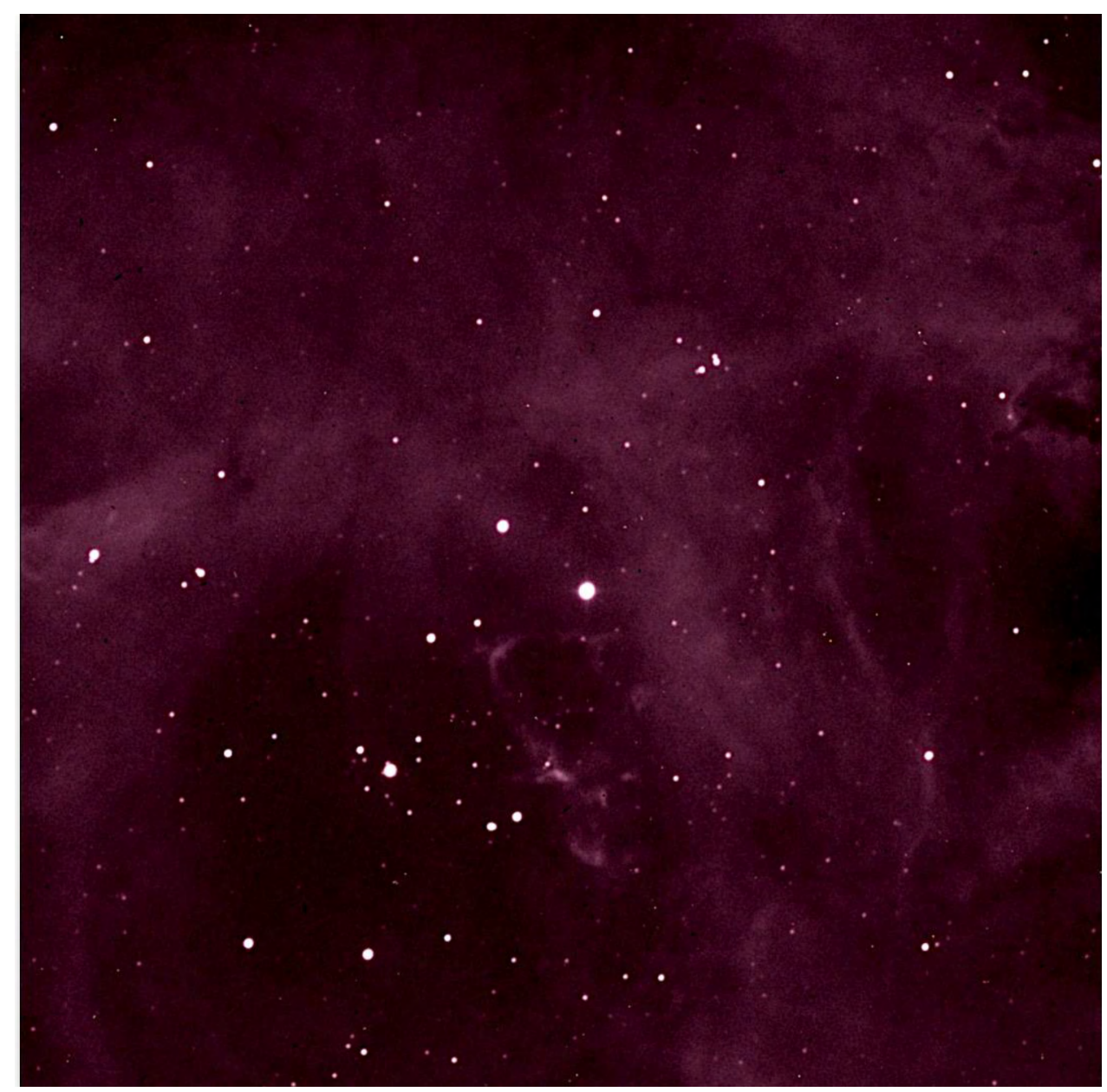

Figure 2. The Rosetta Nebula taken with the R, G and B filters and 180-sec exposures 
Galaxy M81 or Bode's Nebula, was imaged in the constellation Ursa Major and was cataloged by Charles Messier in August, 1779. This image is an LRGB composite of 180-second exposures.

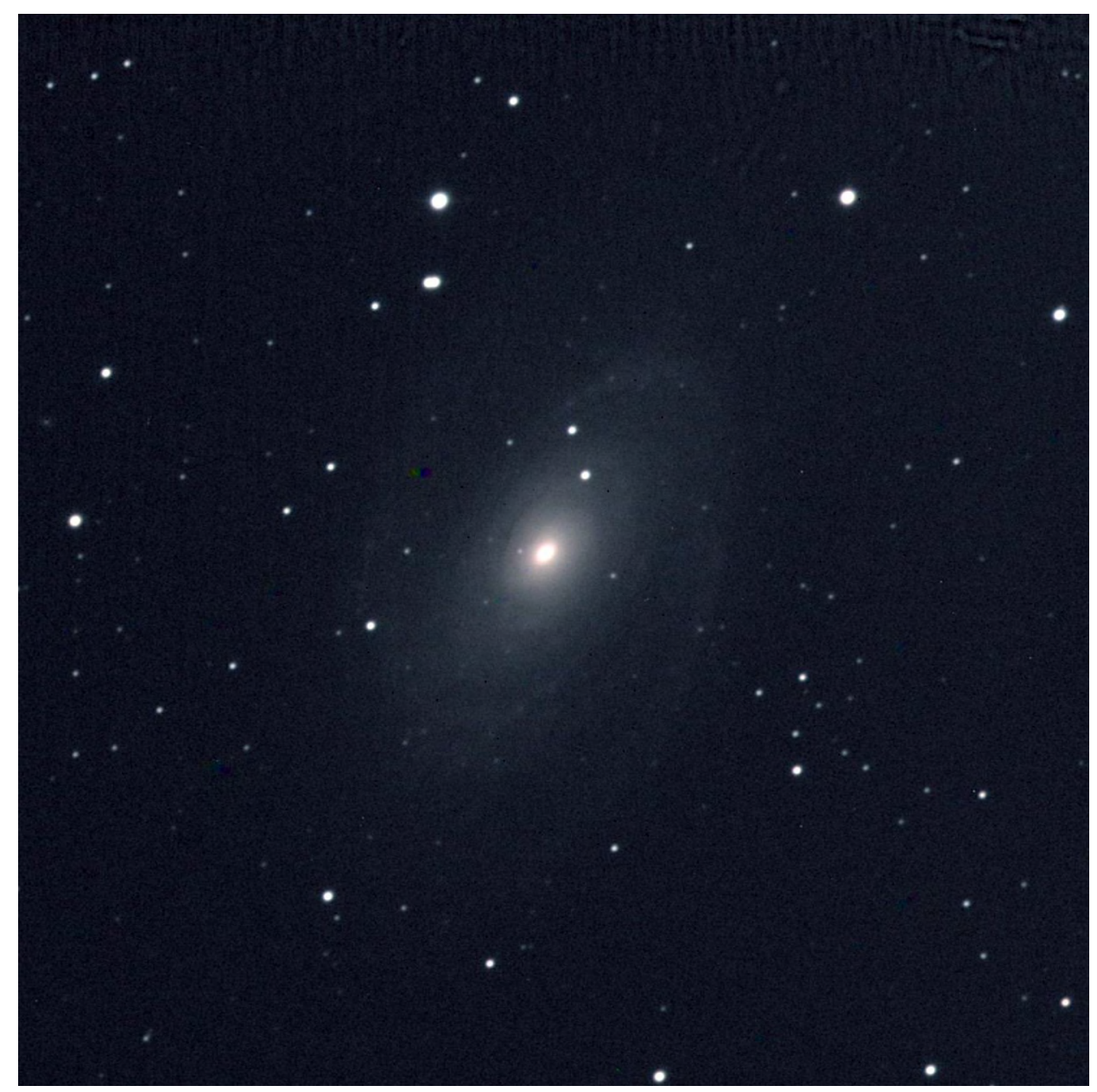

Figure 3. Galaxy M81 or Bode’s Nebula 
Similarly, Galaxy M82 is seen here and is just outside the field of view when centrally focused on M81 by the CDK 24-inch telescope. This galaxy is known as the "Cigar Galaxy" and was also discovered by Messier in the late summer of 1779.

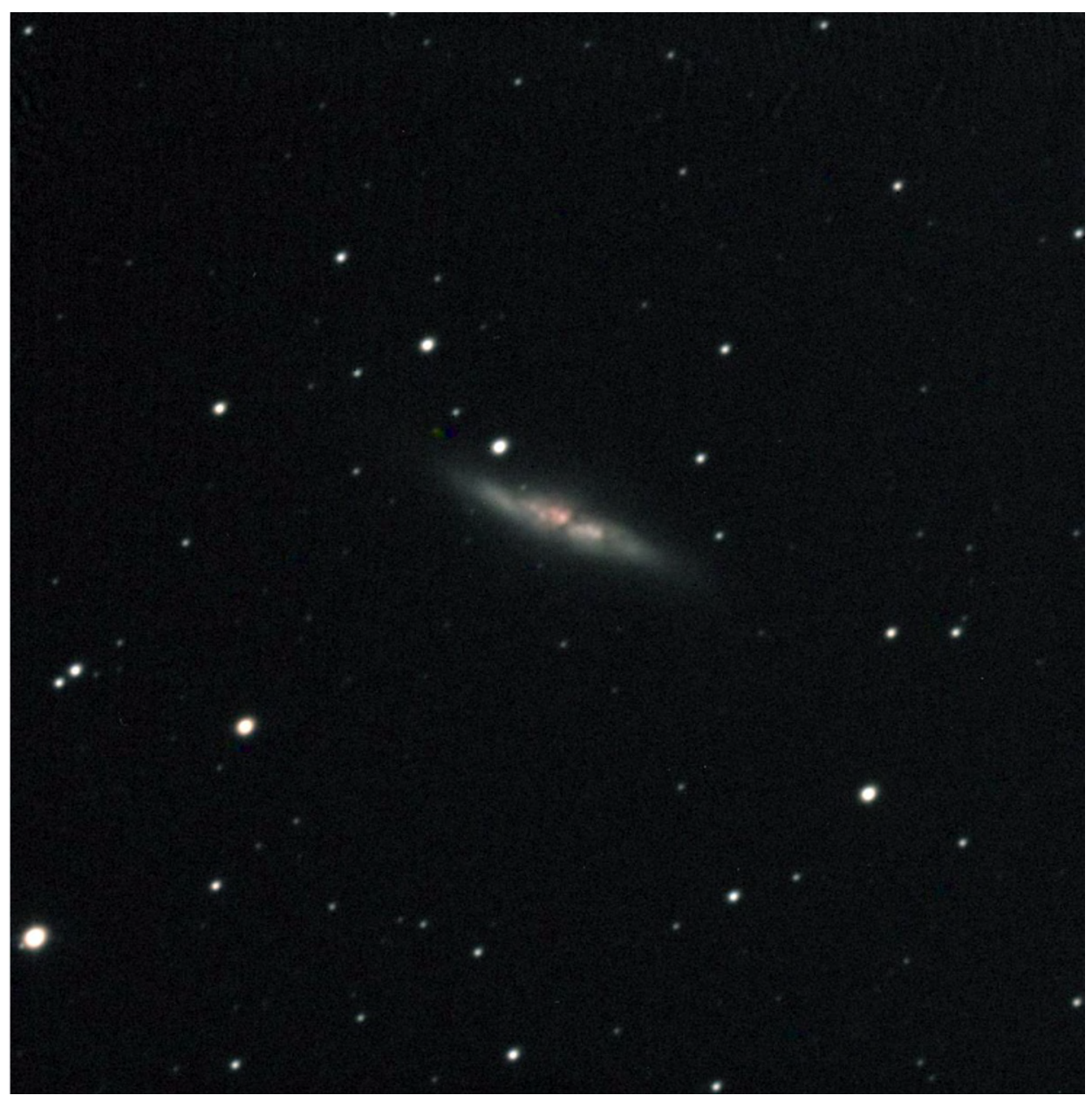

Figure 4. Galaxy M82, also known as the "Cigar Galaxy" 
The Wizard Nebula, also known as NGC 7380 was one of the first color images composed on the APUS observatory from the CDK 24 inch telescope. It took $\mathrm{R}, \mathrm{G}$ and $\mathrm{B}$ color filters at 180 -second exposures.

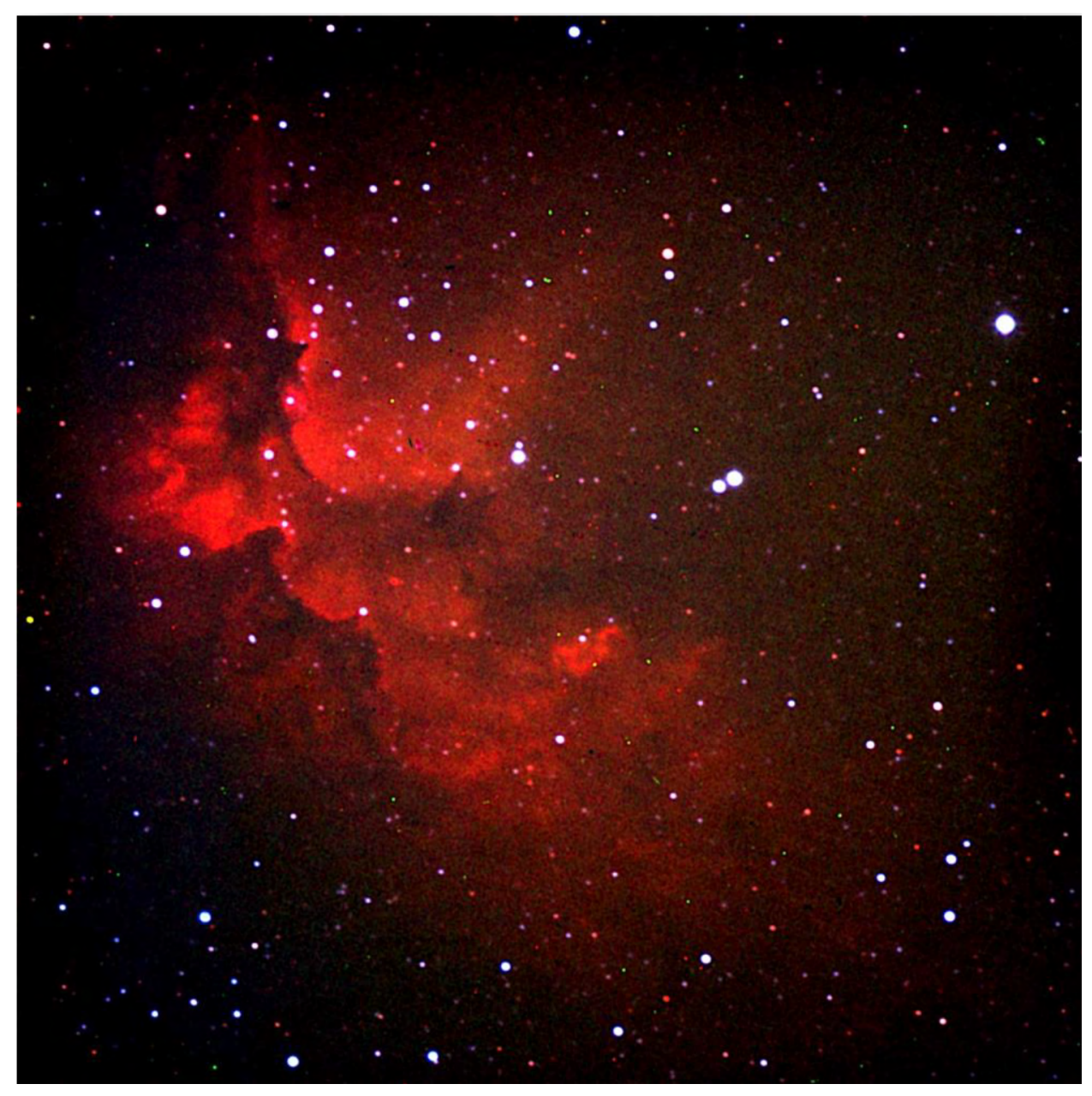

Figure 5. The Wizard Nebula, also known as NGC 7380 
Finally, the emission nebula NGC 281 using the LRGB color filter imaging suite was used in the stacked resultant pictures seen below.

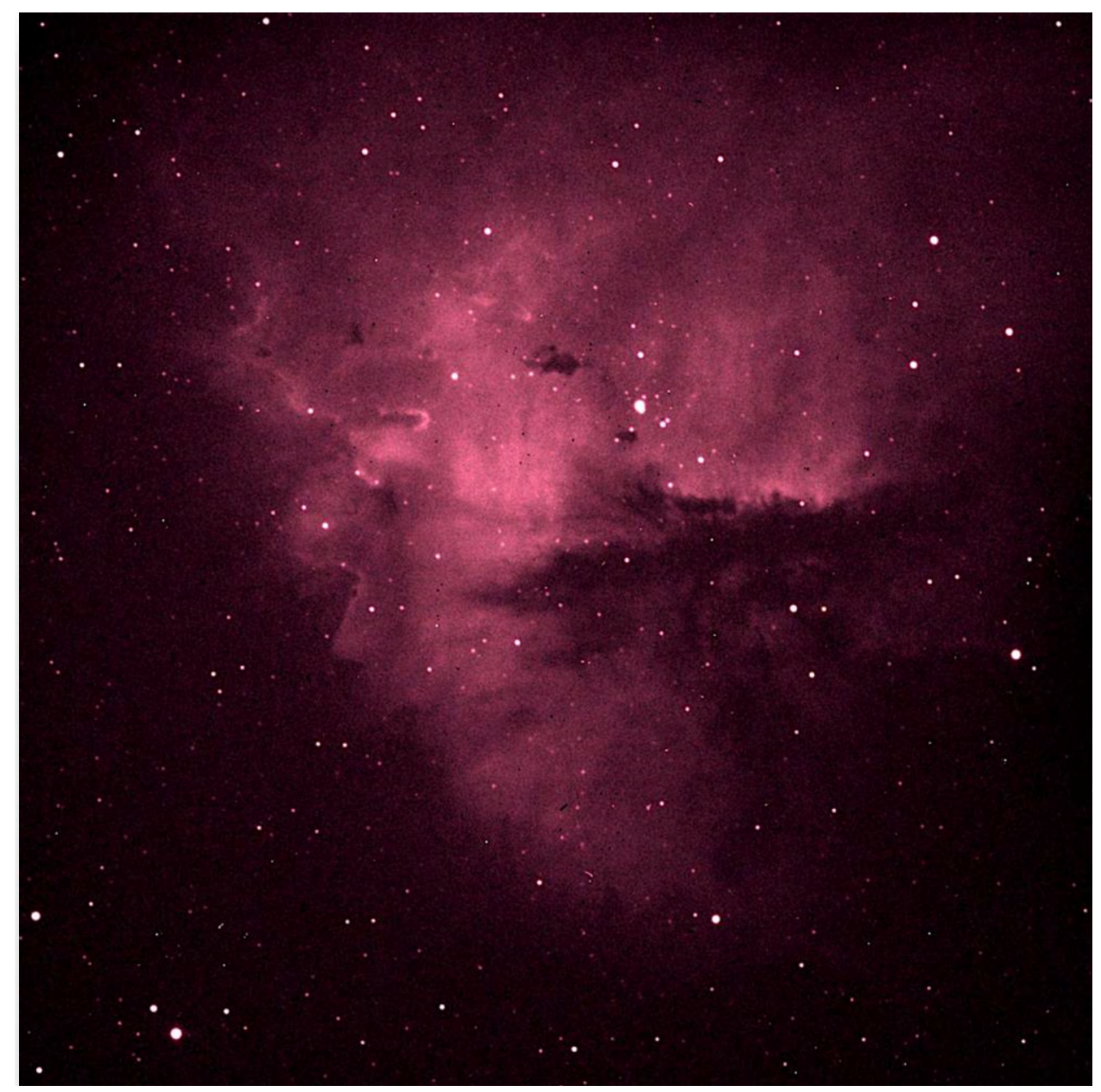

Figure 6. Emission Nebula NGC 281

The APUS observatory has become a great resource for the university's research capabilities and continuing efforts to expand the space studies program to better meet the needs of students, faculty and the science community as a whole. Situated higher than its surroundings, the observatory markedly signifies the vision and goals of APUS, to keep reaching toward the stars and to empower all who use its telescopes. 